\title{
Modeling Radio Channels for CSMA-MAC based Wireless Sensor Networks
}

\author{
Mrutyunjaya Panda \\ Dept. of Electronics and Instrumentation Engineering \\ Gandhi Institute of Engineering and Technology, \\ Gunupur, Orissa, India.
}

\author{
Manas Ranjan Patra \\ Department of Computer science \\ Berhampur University, \\ Orissa, India.
}

\begin{abstract}
Wireless Sensor Network (WSN) has been identified as one of the most important emerging technologies for the $21^{\text {st }}$ century to monitor environments, and have a lot of applications include warfare, smart homes and rescue. This paper presents an insight to the wireless sensor networks using CSMA-based media access control protocol (MAC) with various performance measures to evaluate the efficacy of the model. We use MATLAB based PROWLER to design our simulated WSN model with Normal Radio Model, Radio Model with SINR, and Radio Model using Rayleigh Fading for Spanning tree and geographical angle based routing. The simulation results show that the simulator under valuation can effectively approximate the behavior of WSN in terms of a number of packet sent and received and detected collisions.
\end{abstract}

\section{General Terms}

Computer - Communication Networks, Wireless Communication, Network Protocols

\section{Keywords}

Wireless sensor networks, radio channel, routing

\section{INTRODUCTION}

Wireless sensor networks (WSNs) have become a hot research topic in recent years. A WSN is composed of hundreds or even thousands of small, cheap sensors nodes which communicate with one another wirelessly. Sensor nodes typically do not have very much computational power, limiting the kinds of networking protocols and security mechanisms they can employ. As WSN consists of so many nodes, which are to deployed in a hostile environment, replacing batteries is not feasible. Sensor nodes must therefore survive on the small amount of energy in the battery that are deployed with which is usually about 6ampreHours [1]. This creates a need to conserve energy.

Sensor networks are the key to gathering the information needed by smart environments, whether in home, shipboard, buildings, industrial, transportation, system automation etc. It has also been found that WSN may be useful during terrorist and guerrilla warfare countermeasures which can be deployed in aircrafts with self organizing capabilities in wireless environment [2-3]. Further, WSN must be fast, easy to install and maintain.

The sensor networks operates in a distributive environment called middleware over wireless communication protocols, which should be fault tolerant, adaptive because of the dynamic behavior of the considered network topology. The used protocols for WSN are usually very simple compared to wired networks because of the limited resources of the sensor nodes. The wireless sensors or intelligent sensors are a compact device with its own power source, comprising of a processing unit which is usually a small microprocessor, a communication unit and the sensor itself.

In this paper, we use various routing mechanism along with many variants of radio channel that includes signal to interference noise ratio (RCSINR), Rayleigh fading (RCRF) and radio channel power (RCP) apart from the basic radio channel (RC) are proposed in order to build a wireless sensor network model. All our simulations are carried out in PROWLER, a MATLAB based WSN simulator.

The rest of the paper is organized as follows. Section 2 presents a summary of the related work done in this field of research. A brief description about the Berkeley motes and Tiny OS is presented in Section 3. Section 4 presents the radio channel modeling of our proposed research. Experimental results and discussion are provided in Section 5. Finally, we conclude the paper with some future scope in Section 6.

\section{RELATED WORK}

The authors proposed a contention based MAC protocol (S-MAC) explicitly for wireless sensor networks [4]. In this, they observed that their proposed method achieves efficient low energy consumption along with good scalability and collision avoidance capability. In [5], the authors introduced ER-MAC (Energy and Rate), an energy aware MAC protocol using TDMA (Time division multiple access) and found that it possesses the natural ability of avoiding extra energy wastage. In this, they use the concept of periodic listen and sleep, in the sense that a sensor node switches off its radio and goes into a sleep mode only when it is in its own time slot and does not have anything to transmit. Sahoo et al. [6] presents a TDMA based MAC protocol that can provide delay guarantee. It is also pointed out in [6] that their proposed approach RT-MAC takes less time relativity by reutilizing the connection channel between two successive channel accesses of a sensor node. However, the problem lies in performing a lot of calculations that could exhaust the sensor node itself in some situations. In [7], the authors provide one of the first works with experimental measurements and but they do not provide any explanation of their findings. Recently, Reijer et al. [8] presents empirical results of extensive link layer measurements with the eyes nodes, but they do not provide any channel model. The problem of convergecast in adhoc geometric networks is considered in [9], where the authors assume that a transmitting node is capable of detecting collisions within its transmission range and the duration of the time slot was long enough to allow transmission of multiple packets in one time slot. The authors propose some practical considerations for wireless sensor network 
algorithms and provide pointers to remedy the lack of connection [10].

\section{THE BERKELEY MOTES AND WIRELESS SENSOR NETWORK MODEL}

The Crossbow Berkeley motes are one of the most versatile wireless sensor network devices on the market for prototyping purposes. Crossbow manufactures three types of Mote processor radio module as: MICA [MPR 300] which is the first generation mote, MICA2 [MPR 400] and MICA2-DOT [MPR 500] (second generation). These motes are found suitable especially for surveillance networks intended for human and vehicles. Since all sensor nodes (motes) in a network can act as base stations, the network can therefore be self configured with multi-hop routing capabilities. The operating frequency of Berkeley motes are in ISM band, either $916.5 \mathrm{MHz}$ or $433 \mathrm{MHz}$, with a data rate of 40kilobits per seconds, and having a range of 30 feet to 100 feet. In this paper, we use Berkeley field nodes (motes) which contain an 8-bit microcontroller, a $916.5 \mathrm{MHz}$ radio and several interchangeable sensors. Each mote has a low power microcontroller with speed of $4 \mathrm{MHz}$, a flash memory with 128 kilobytes with SRAM and EEPROM of $4 \mathrm{~KB}$ each. These tiny units have simple local micro-threading distributed operating systems called TinyOS, developed by US Berkeley. A typical sensor mote is shown below in Figure 1.

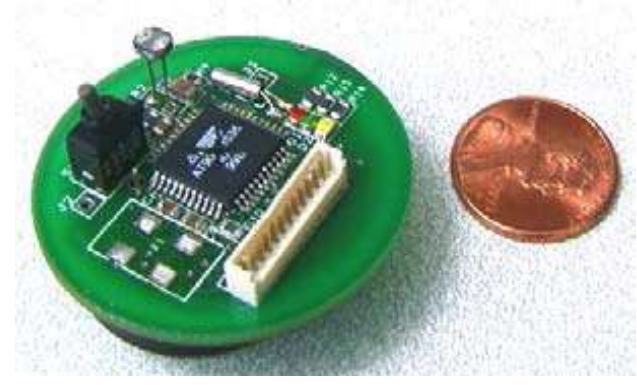

Figure 1. A Berkeley Mote

A general model for wireless sensor network simulation at a network-wide scale is provided in Figure 2 below.
* Each node is a physical device monitoring a set of physical variables. The nodes communicate to each other through a common radio channel. Internally, a protocol stack with an additional topology component to control communications and node coordinates respectively to position the nodes in 2or 3- dimensional world. A typical WSN model contains from few to several thousands of nodes depending on the application and deployment scenario into consideration.

* Environment plays a vital role in modeling a WSN in that it models the generation and propagation of events that are sensed by the nodes and perform sensor actions by communicating among the nodes deployed in the network. The events are generally a physical magnitude as sound or temperature.

* Finally, the agents are the event generators for the sensor nodes. This is useful when its behavior can be implemented independently from the environment, like a mobile vehicle or else the environment can generate the events on its own.

\section{PROPOSED METHODOLOGY}

The protocols used for wireless sensor networks are broadly divided into two categories. They are:

- TDMA (time division multiple access) based MAC protocols, in which the medium is divided into time slots. In this, the nodes are having knowledge of their individual time slots of entering the medium and perform the intended operation. Example, ALOHA [11].

- CSMA (Carrier sense multiple access) based MAC protocols, where the wireless nodes compete to enter into the wireless medium for connectivity and finally, the winner node reserves the medium for itself until it finishes its operation. Example fall in this category include, 802.11 [4], S-MAC [4], etc.

In this paper, we use MAC protocols using CSMA for various radio channel possibilities with span tree application.

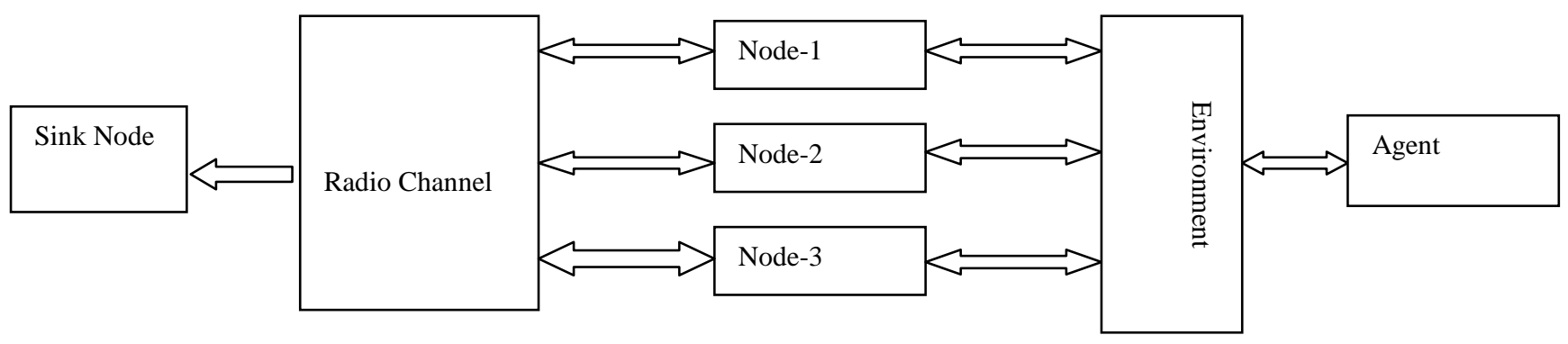

Figure 2. Model for WSN simulation

* The sink nodes are the nodes that receive the data from the net and process it, which may further interrogate the sensors about an event of interest. The use of sink nodes largely depends on the simulation environment.

* The radio channel characterizes the propagation of radio signals among the nodes in the used network.

\subsection{Radio Channel propagation Models}

The radio channel (RC) propagation model determines the strength of a transmitted signal at a particular point of the space for all transmitters in the system, based upon which conditions of the signal receptions for the receivers can be evaluated and detection of collisions can be obtained. In this scenario, the strength of the signal from transmitter to receiver decreases with 
distance as a deterministic propagation function as well as by random disturbances by considering fading effect, time varying nature of the signal strengths, and other transmission errors, etc.

\subsection{Signal reception and Collision}

In this paper, we use four models for the simulation of the wireless sensor network.

Model-1 uses a normal radio channel (RC). In this, the signal is received if the signal strength is greater than a reception threshold. The channel is sensed idle if there is no signal to be received or collision, if two transmitted signals overlap each other in time and then could be received. This model is simple and fast, but not very accurate.

Model-2 builds a radio channel model with signal to interference and noise ratio (RC-SINR) into consideration, with a receiver noise variance parameter. In this case, the channel is considered ideal if the total signal strength is smaller than an idle limit, which depends on the noise variance of the receiver. On the other hand, the signal is received if the SINR at the receiver is greater than specified threshold during the whole length of the transmission. This model provides more accurate results at a somewhat slower speed.

Model-3 uses a fading radio channel using Rayleigh Fading (RC$\mathrm{RF}$ ) that describes a scenario where the motes do not have direct line of sight (Rician Fading).

Model-4 uses power of a radio channel (RC-Power) to design a wireless sensor network.

\section{EXPERIMENTAL RESULTS AND DISCUSSIONS}

We perform our simulation model to design a wireless sensor network and evaluate its performance using PROWLER 1.25, a probabilistic events-driven simulator [12]. One of the main advantages of the Prowler framework is its ease to use, for its MATLAB environment. It provides a customizable GUI for entering network parameters (topology type, number of motes) and animation. There are three modules present in the prowler. The first module is the main module which handles event called by the user (i.e. set clock, send packet) or are fired by the other events (i.e. packet_received, clock_tick, timer_fired). The second one is the radio module that handles both the MAC-layer model and the radio propagation model. Finally, the third module is the application module provides access to user generated events.

\subsection{Routing protocols Used}

\subsubsection{Angle Routing}

This paper proposes a routing protocol based on the angles (directions) of the adjacent sensor nodes. Each pair of nodes that form a hop should ideally be moving in the same or similar direction, so the connection between the source and the destination will consist of a series of nodes that are moving in a similar direction.

\subsubsection{Spanning Tree}

It is a link management protocol that provides path redundancy while preventing undesirable loops in the network. Spanning trees are widely used in many distributed applications like sensor networks. In this, only one active path can exist between two stations for an Ethernet network work properly, where as multiple active paths between stations cause loops in the network. Further, in this type of applications, the routing of data and messages takes place on a virtual overlay network that is constructed on top of the underlying physical network. Such overlay network plays a measure role in addressing the efficiency of the sensor network. More details about this can be found in [13].

\subsection{Pursuer Evader Games}

The Pursuer Evader Game (PEG) [14] has been a standard test bed for sensor networks, where sensors are deployed in a regular grid with random offsets. A pursuer is a vehicle that is going to track the evader down, where the evader is a car whose movement can be detected by the sensor nodes. In this process of communication, the problem is to route the packets sent out by the sensor nodes who detected the evader to the mobile pursuer.

In this paper, we use a network of 10x10 sensor grid with small random offsets. The network is built with 100 nodes uniformly spread on a square of $200 \mathrm{~m}$ by $200 \mathrm{~m}$. The transmit signal strength is set to 1 , and the maximum radio range is about $3 \mathrm{~d}$ for a dense network, where $d$ is the standard distance between two neighbor nodes in the grid. In this source starts at the center and the sink starts at a corner. The source is dynamic in nature, changing from node to node, following the movement of the evader, and the sink is mobile. The average speed of the evader and pursuer is set to $0.2 \mathrm{~d} / \mathrm{s}$. An instance of the connectivity of such a wireless sensor network is shown in the right side of the Figure 3. The radio data rate is $40 \mathrm{kbps}$ and each packet has 960 bits. The application sends out one packet per second from the sources to the destination. Finally, the results are computed on the average of 10 random runs to evaluate the performance of the model.

\subsection{Performance Metrics}

We use the following routing performance metrics for the comparison of the different routing protocols applied in the sensor network building process with various radio channel considerations.

4 Latency: It is a measure of time delay that occurs during transmission of the packet (messages) from source to the sink. Latency is used here instead of number of hops because latency is caused not only by the number of hops but also by the length of transmission queues, the random delays at the MAC layer and delay addition in routing algorithms to avoid collisions.

4 Success Rate or Delivery Ratio: It is measured as the ratio between total numbers of data packets received at all sinks and the total number of data packets generated at all sources.

4 Energy efficiency: It is defined to be the ratio between the total number of received data packets and the total number of packets transmitted in the network. This is evaluated by considering that transmission of a data packet consumes $20 \mathrm{~nJ} /$ packet while reception uses 3 onJ/packet but the actual cost may be varied based on the radio and environment used for the analysis. The module stops the simulation when a first mote dies.

\subsection{Simulation Results}


We use Span tree and angle routing of PROWLER 1.25 with different radio channel considerations with alpha $=0.7854, \mathrm{p}=0.5$. The results obtained after performing the simulation with Spanning tree routing and angle routing with various radio channel consideration, as discussed earlier are shown in Figure 3 to Figure 11. Finally, comparison to various methodologies adopted in this proposed work is presented in Table 1. From all the results, it is evident that the proposed approaches provide very small latency in time with high delivery ratio, energy efficient and faster simulation while building the wireless sensor network model.

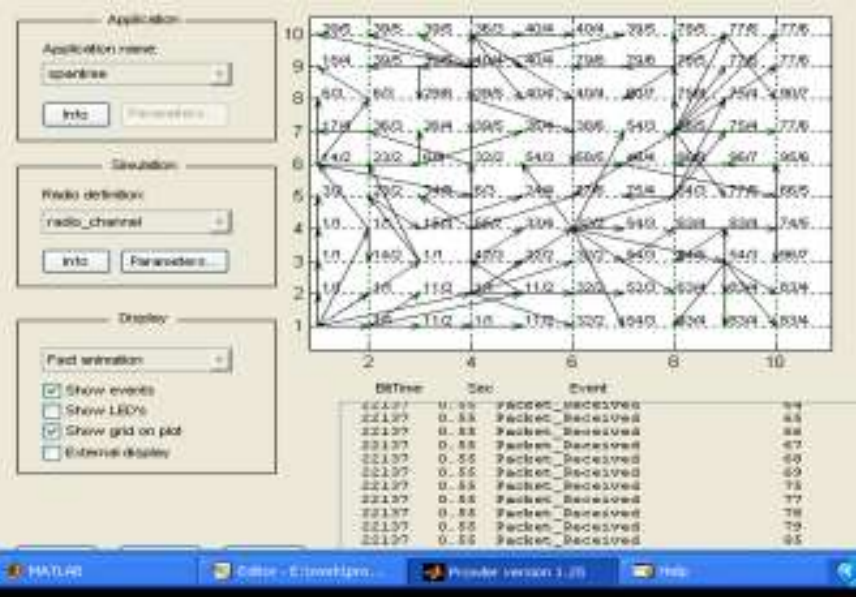

Figure 3. Span tree with RC

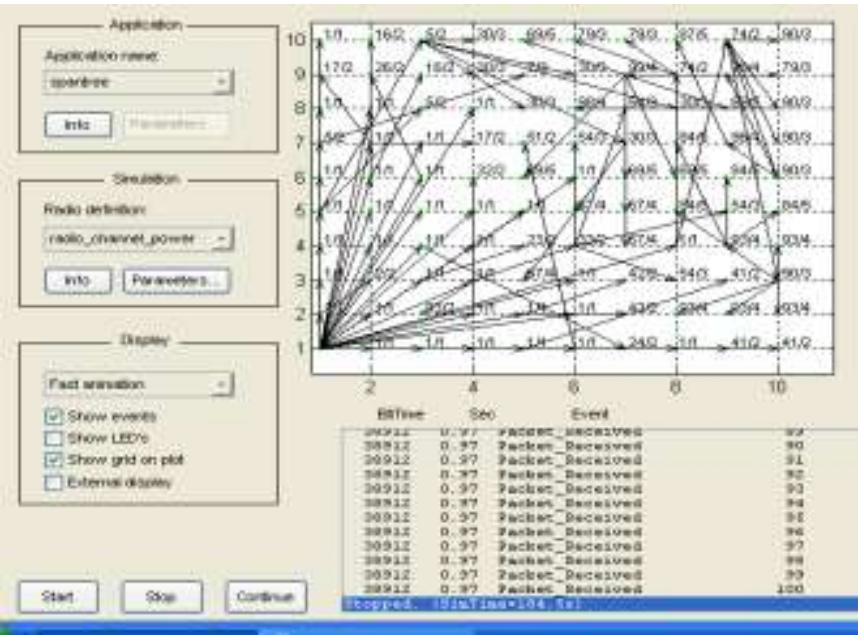

Figure 4. Span Tree with power radio channel

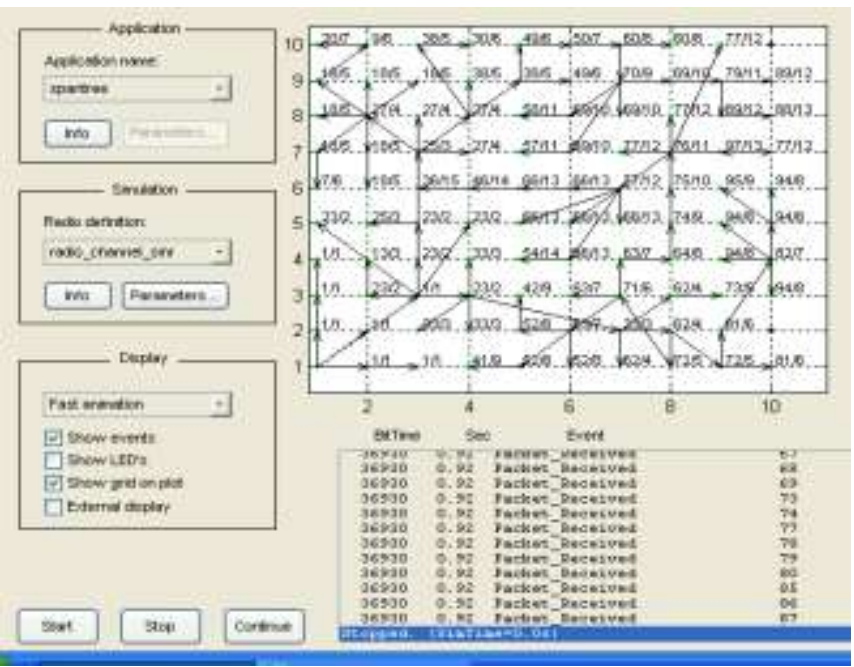

Figure 5. Span Tree with Radio Channel with SINR

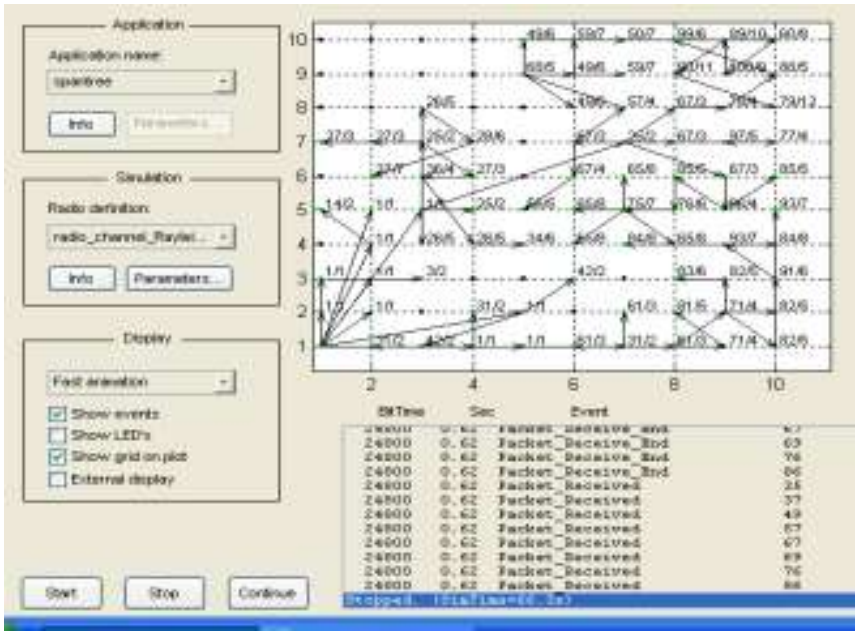

Figure 6: Span tree with radio channel with Rayleigh fading

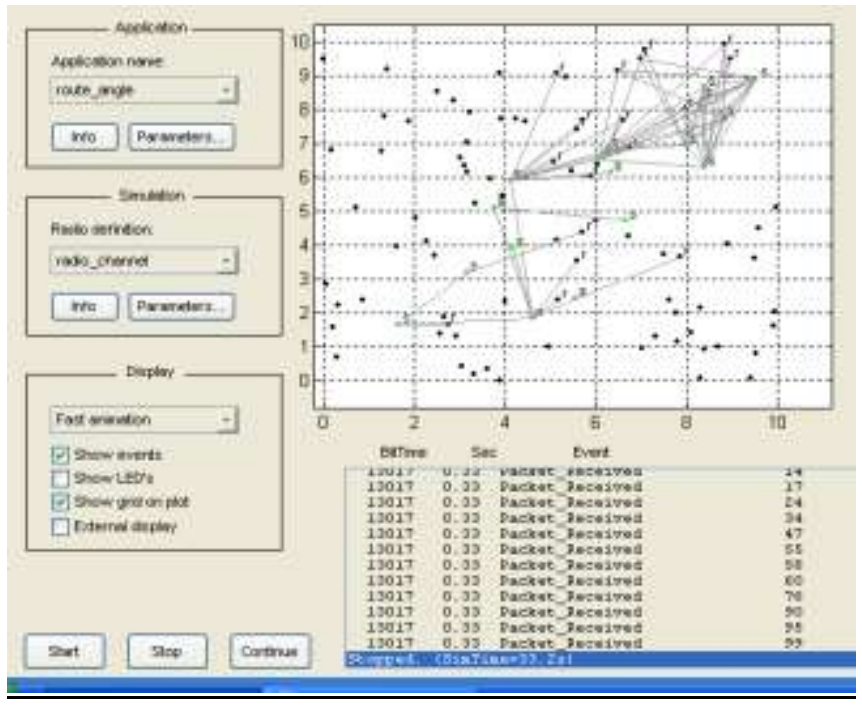

Figure 7 Angle routing + RC with alpha $=0.7854, p=-.5$ 


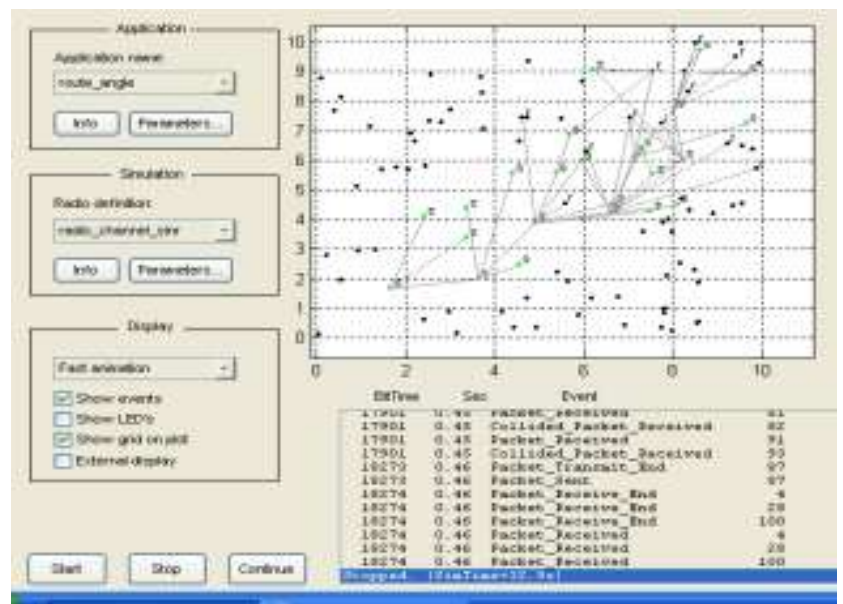

Figure 8 . Angle routing + SINR

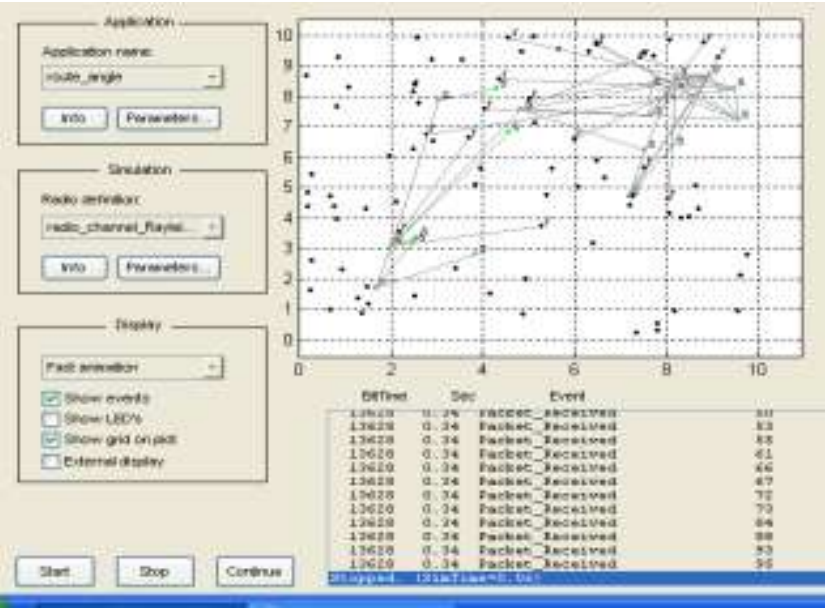

Figure 9. Angle routing $+\mathrm{RF}$

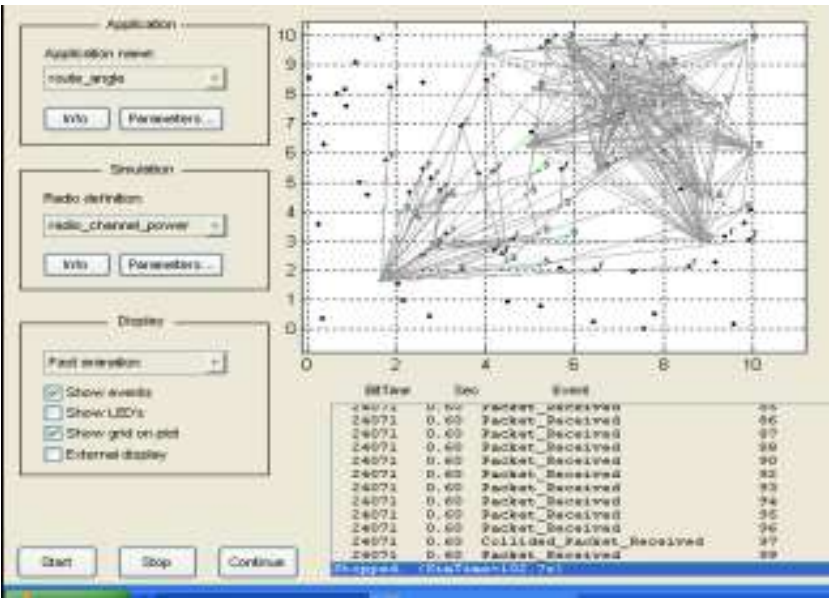

Figure 10. Angle routing +Power

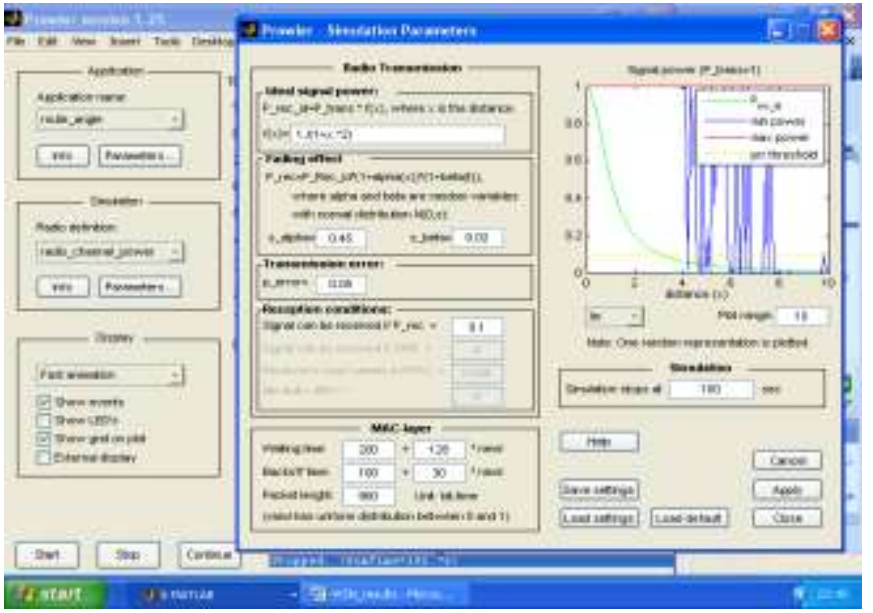

Figure 11. Radio channel model

Table 1. Performance evaluation of various radio channels in WSN

\begin{tabular}{|l|l|l|l|l|l|}
\hline Metrics $\longrightarrow$ & $\begin{array}{l}\text { Latency in } \\
\text { Seconds }\end{array}$ & Success Rate & $\begin{array}{l}\text { Energy } \\
\text { Efficiency }\end{array}$ & $\begin{array}{l}\text { Throughput in bits } \\
\text { per second }\end{array}$ & $\begin{array}{l}\text { Simulation Time } \\
\text { in Seconds }\end{array}$ \\
\hline SpanTree+RC & 0.024 & 5.484 & 8.227 & 1096.08 & 121.8 \\
\hline SpanTree+RC+ Power & $6.7 * 10^{-3}$ & 14.01 & 21.015 & 1498.71 & 184.5 \\
\hline SpanTree+RC+SINR & 0.024 & 2.847 & 4.27 & 321.3 & 30.2 \\
\hline SpanTree+RC+RF & 0.024 & 2.088 & 3.133 & 292.32 & 66.3 \\
\hline Angle Routing + RC & 0.024 & 15.176 & 22.765 & 955.55 & 33.2 \\
\hline $\begin{array}{l}\text { Angle Routing +RC + } \\
\text { power }\end{array}$ & 0.024 & 29.4 & 44.1 & 1881.17 & 102.7 \\
\hline $\begin{array}{l}\text { Angle Routing + RC + } \\
\text { SINR }\end{array}$ & 0.024 & 9.21 & 13.812 & 549.75 & 32.9 \\
\hline $\begin{array}{l}\text { Angle Routing +RC + } \\
\text { RF }\end{array}$ & 0.024 & 8.833 & 13.25 & 559.86 & 0.0 \\
\hline
\end{tabular}




\section{CONCLUSION AND FUTURE SCOPE}

In this paper, we have presented the framework of Spanning tree and Angle routing protocol in a CSMA-MAC application with different radio channel consideration in order to build a wireless sensor network model. We have evaluated our proposed methodologies using five performance metrics to understand the efficacy of the built network. However, we need to develop and test many other routing protocols in this scenario in future to understand the issues relating in designing a robust wireless sensor network.

\section{REFERENCES}

[1] Michael Brownfield, Wireless sensor network denial of sleep attack. In: proc. Of the 2005 IEEE workshop on information assurance and security, United states military academy, West point, NY.

[2] F. Zhao, and L. J. Guibas. Wireless Sensor networks: An Information processing approach. Morgan Kaufmann publisher, 2002 .

[3] I. F. Akyildiz, W. Su, Y. Sankarasubramaniam, and E. Cayirci. Wireless Sensor Netwroks: A Survey. IEEE Transactions on Systems, man and Cybernatics, Part-B, Vol. 38, No. 4, pp. 393-422, 2002.

[4] W. Ye, J. Heidemann and D. Estrin. An energy efficient MAC protocol for wireless sensor network. USC/ISI Technical report, ISI-TR-543, September, 2001.

[5] R. Kannan, R. Kalidini and S. S. Iyengar. Energy and rate based MAC protocol for wireless sensor networks. SIGMOD record, Vol. 32, No. 4, December 2003.

[6] A. Sahoo and P. Baronia. An energy efficient MAC in wireless sensor networks to provide delay guarantee. $15^{\text {th }}$ IEEE workshop on Local and Metropolitan Area Networks, LANMAN 2007.

[7] J. Zhao and R. Govindan. Understanding packet delivery performance in dense wireless sensor networks. In: Proc. of ACM SnSys 2003, Los Angeles, California (USA), November 2003.

[8] N. Reijers, G. P. Halkes and K. G. Langendoen. Link layer in sensor networks. In: proc. of IEEE Intl. conference on Mobile Adhoc and Sensor systems 2004, Florida (USA), October 2004.

[9] A. Kesselman and D. Kowalski. Fast distributed algorithm for convergecast in adhoc geometric radio networks. In: Proc. of the $2^{\text {nd }}$ Annual conference on wireless on demand network systems and services, 2005.

[10] G. Halkes and K. Langendoen. Practical considerations for wireless sensor network algorithms. Wireless sensor networks, Vol. 2, 2010, pp. 441-446.

[11] J. K. Park, W. C. Shin and J. Ha. Energy aware pure ALOHA for wireless sensor networks. IEIC Transaction Fundamentals, Vol. E89-A, No. 6, June 2006.

[12] G. Simon, Prowler: Probabilistic wireless network simulator. Institute for software integrated systems (ISIS). http://www.isis.vanderbilt.edu/projects/nest/prowler.

[13] S. A. Attarde, L. L. Ragha and S. K. Dhamal. An enhanced spanning tree topology for wireless sensor networks. International Journal of computer applications, Vol. 1, No. 19, 2010, pp. 46-51.

[14]Y. Zhang and M. Fromherz. A robust and efficient flooding based routing for wireless sensor networks. Journal of interconnection networks. Vol. 7, No. 4, 2006, pp. 549-568. 